\title{
Objective Bayesian analysis of "on/off" measurements
}

\author{
Diego Casadei ${ }^{1}$ \\ School of Engineering, FHNW, Bahnhofstrasse 6, 5210 Windisch, Switzerland \\ diego.casadei@fhnw.ch
}

\begin{abstract}
In high-energy astrophysics, it is common practice to account for the background overlaid with the counts from the source of interest with the help of auxiliary measurements carried on by pointing off-source. In this "on/off" measurement, one knows the number of photons detected while pointing to the source, the number of photons collected while pointing away of the source, and how to estimate the background counts in the source region from the flux observed in the auxiliary measurements. For very faint sources, the number of detected photons is so low that the approximations which hold asymptotically are not valid. On the other hand, the analytical solution exists for the Bayesian statistical inference, which is valid at low and high counts. Here we illustrate the objective Bayesian solution based on the reference posterior and compare the result with the approach very recently proposed by Knoetig (2014), discussing its most delicate points. In addition, we propose to compute the significance of the excess with respect to the background-only expectation with a method which is able to account for any uncertainty on the background and is valid for any photon count. This method is compared to the widely used significance formula by Li\&Ma (1983), which is based on asymptotic properties.
\end{abstract}

Subject headings: gamma-rays: general - methods: statistical

\section{Introduction}

In a counting experiment, the detector response to a trigger signal is saved, whenever at least one among (possibly many) different conditions is satisfied. The trigger requirements are defined in such a way to select interesting "events" and operate the detector in the most efficient way. Counting experiments are widespread in high-energy physics and astrophysics, and sometimes have to deal with very low event rates. This is the case, for example, when one tries to observe a very faint gamma-ray source with a space experiment, or when the goal is to detect the excess of counts corresponding to a new particle created by the collisions produced by underground particle accelerators.

When only few events are collected, the asymptotic espressions which can be used with high

\footnotetext{
${ }^{1}$ Visiting Scientist, Department of Physics and Astronomy, UCL, Gower Street, London WC1E 6BT, UK
}

count rates can not be adopted any more. Instead, it is of great importance to study the correct statistical model without simplifying assumptions which could invalidate the result. For counting experiments, it is commonly assumed that the integer number $n \geq 0$ of observed events follows the Poisson distribution:

$$
\operatorname{Poi}(n \mid a)=\frac{a^{n}}{n !} e^{-a}
$$

where the real value $a \geq 0$ is the Poisson parameter, which coincides with the expected number of events and with the variance: $E[n]=V[n]=a$.

Realistic measurements always involve some degree of "background" counts, due to noninteresting events which satisfy (hopefully, but not always, with low probability) some trigger condition. We assume that the counts from the source of interest and those from the background are independent Poisson variables. A well known property of the Poisson distribution is that the sum of two independent variables is again Poisson 
distributed, with parameter given by the sum of the respective expectations:

$$
P(n \mid s, b)=\operatorname{Poi}(n \mid s+b)=\frac{(s+b)^{n}}{n !} e^{-(s+b)}
$$

where the expected number of events from the source $s \geq 0$ is the parameter of interest, and the background contribution $b \geq 0$ is the nuisance parameter ( $n$ is integer, whereas $s$ and $b$ are real numbers).

In high-energy astrophysics, it is common to estimate $b$ with the help of auxiliary measurements, obtained by pointing the detector off the source 1 In this case, it is assumed that the source of interest does not contribute to the observed $k$ counts, such that one has a simple Poisson process:

$$
\operatorname{Poi}(k \mid B)=\frac{B^{k}}{k !} e^{-B}
$$

where $B \geq 0$ is the expected (background-only) photon count in the region off the source. By knowing the details (like the area on the sky and the exposure time) of the source and off-source regions, it is possible to relate the expected counts from the background alone in the two regions: $b=\rho B$, where $\rho$ is a constant, assumed to be perfectly known (i.e. with negligible uncertainty compared to $B)$.

In summary, the statistical inference about this "on/off" measurement makes use of the observed counts $n$ and $k$ in the source and off-source regions, of the known proportionality $\rho$ between the expected background fluxes in the two regions, and of the Poisson models (1) and (2) for the two measurements.

Recently, Knoetig (2014) (MK2014 hereafter) summarized the previous approaches to the on/off inference problem and proposed an objective Bayesian solution which consists of two different steps. First, it is checked whether the number $n$ of events in the source region is too high to be comfortably attributed to the background alone. If this is the case, one rejects the "null hypothesis" (background only) and claims a successful observation of the source. Next, the source intensity $s$ is estimated with the help of the auxiliary

\footnotetext{
${ }^{1}$ In high-energy physics, the background is estimate by looking at "control regions" in which the expected signal is negligible or null. Alternatively, Monte Carlo simulations are used to estimate the background in the "signal region".
}

measurement. The good point is that MK2014 finds a (rather complicate) analytic solution to the Bayesian inference problem, in terms of special (Gamma and hypergeometric) functions which are available in many libraries.

The procedure proposed by MK2014 is well motivated and it aims at achieving very desirable goals. However, it is not free from issues. The first source of possible troubles is the proposed prior [eq. (15) of MK2014], which is obtained following the Jeffreys' rule in the bidimensional $(s, b)$ space [called $\left(\lambda_{\mathrm{s}}, \lambda_{\mathrm{bg}}\right)$ in MK2014]. Even though Jeffreys' priors have a number of desirable properties for 1-dimensional problems, it is well known that they behave badly in multidimensional problems [for a recent discussion, see Berger et al. (2013)]. Here we overcome this difficulty by reducing the problem to a 1-dimensional (marginal) model for which an objective prior is known.

One further complication in the procedure proposed by MK2014 is the comparison of the null hypothesis $H_{0}$ that $s=0$ (called "backgroundonly hypothesis" here) against the alternative hypothesis $H_{1}$ that $s>0$ in the target region (the "source+background hypothesis"). In absence of additional information, MK2014 assigns identical (prior) probabilities to the two hypotheses: $P\left(H_{0}\right)=P\left(H_{1}\right)=1 / 2$. They are "nested" hypotheses, in the sense that $H_{0}$ assumes a single value whereas $H_{1}$ allows the source strength to assume any other value in its domain. The fact that the measure of the allowed domain is zero for $H_{0}$ gives troubles when using improper priors[(see for example Bayarri et al. (2008) and references therein].

To overcome the problem, MK2014 fixes the ratio between the arbitrary scale factors of the source and background priors with a procedure relying on the assumption that, when no counts are observed both in the target and off-source regions, the probabilities of $H_{0}$ and $H_{1}$ stay the same. This assumption is questionable, as counting zero events is not the same as performing no measurement. If the outcome is $k=0$ in the auxiliary region, we learn that the background in the source region is very small (possibly negligible), and this may imply a better sensitivity (see Appendix A for more details). In addition, if we count $n=0$ events in the source region, we know that there is no background contribution in this 
outcome and also that there is no count due to the source (which makes it easy to get an upper bound to its intensity). Thus, intuitively one may think that the absence of counts in the target region decreases the probability that a source is actually there, hence increasing $P\left(H_{0}\right)=1-P\left(H_{1}\right)$, contrarily to MK2014. Although this way of thinking might also be criticized, what matters here is that the assumption, that measuring zero counts in both regions does not update our degree of belief about the validity of both the background-only and the source+background hypotheses, is questionable and can not be taken as a basis for the hypothesis test.

In this paper, we present the objective Bayesian approach based on the analytical solution of model (1) in the framework of the Bayesian reference analysis (Bernardo 2005a) obtained by Casadei (2012) (DC2012 hereafter). This solution is based on the "reference prior" corresponding to the model given in equation (1), which has a solid formal justification and does not suffer from the problems of multidimensional Jeffreys' priors. In addition, frequentist coverage studies have been carried on by DC2012 and show a good average agreement between the posterior probability and the coverage (exact agreement is not possible, as this is a discrete problem).

The reference prior computed by DC2012 may be coded in any programming language $(\mathrm{a} \mathrm{C}++$ version is adopted here), and is also available in the Bayesian Analysis Toolkit (Caldwell et al. 20092 . For the users of other analysis frameworks and/or programming languages, it may be useful to know that simple approximations also exist, which are even quicker to compute (Casadei 2014).

To illustrate the application of the objective Bayesian approach of DC2012 and compare to MK2014, our solution will be applied in a simplified way (i.e. in a single-step procedure) to the same Gamma-Ray Burst (GRB) data listed in table 1 of MK2014. The marginal reference posterior probability density of the source strength $s$ will be estimated directly, without comparing $H_{0}$ against $H_{1}$. Similarly to MK2014, the posterior distribution for $s$ will be summarized by provid-

\footnotetext{
${ }^{2}$ Few lines of BAT instructions are sufficient to setup and solve the problem: a complete example is provided in the examples/advanced/referencecounting/ directory.
}

ing its mode, i.e. the most probable value or peak position, together with Highest Posterior Density (HPD) intervals, which are the narrowest intervals covering a predefined posterior probability. Whenever one of such intervals is limited by zero at the left, its right edge automatically provides an upper bound to the source intensity ${ }^{3}$ In this case, it will be assumed that no source was detected, so that the alternative hypothesis of source+background is discarded. In our conservative approach, when the posterior for $s$ suggests a non-negligible intensity we check the statistical significance of the excess of counts with respect to the backgroundonly hypothesis to decide whether to claim a successful source detection or not. This procedure provides results which are essentially equivalent to the two-steps approach involving the comparison of two hypotheses, without the complications arising from the latter in the presence of improper priors (as it is the case for the on/off problem).

\section{Methods}

We have two Poisson models, characterizing a region where no source contribution is expected, eq. (2), and the target region where a source may produce counts in addition to those coming from the background alone, eq. (1). The available data are the number $n$ of photons detected when pointing to the source (called $N_{\text {on }}$ in MK2014), the $k$ off-source counts (called $N_{\text {off }}$ in MK2014), and the ratio $\rho=b / B$ (called $\alpha$ in MK2014) between the background fluxes in both regions. One first has to estimate the background in the target region with the help of the auxiliary off-source measurement. Next one considers the marginal model obtained by integrating over $b$ and finds the reference prior for $s$. Finally, one gets the reference posterior for $s$ with the help of Bayes' theorem.

The auxiliary measurement is analyzed first. The Poisson distribution (2) allows to estimate the off-source background intensity $B$ by means of the

\footnotetext{
${ }^{3}$ It is useful to recall the difference between an upper limit on the intensity which one expects to detect with a predefined probability, characterizing the sensitivity of the detection technique, and an upper bound on the intensity inferred from the actual measurement (Kashyap et al. 2010). Here we are only concerned with the latter. Appendix A provides details about upper limits.
} 
Bayes' theorem:

$$
p(B \mid k) \propto \operatorname{Poi}(k \mid B) \pi(B)
$$

(we omit the proportionality constant, as the latter can be determined by imposing that the integral of $p(B \mid k)$ be one). This equation expresses the posterior probability density function $p(B \mid k)$ of the off-source background intensity $B$ given the $k$ observed counts, in terms of the likelihood function (2) and of the prior density $\pi(B)$.

If we have some prior estimate of the background flux in the off-source region, it is most convenient to represent it with a Gamma distribution (the conjugate prior of the Poisson model):

$$
\mathrm{Ga}(x \mid S, R)=\frac{R^{S}}{\Gamma(S)} x^{S-1} e^{-R x}
$$

with shape parameter $S>0$ and rate parameter $R>0$. In this case, the posterior also belongs to the Gamma family, with new shape and rate parameters $S^{\prime}=S+k$ and $R^{\prime}=R+1$, corresponding to $k$ observed counts 4 . For example, the prior parameters $S, R$ can be fixed with the the method of moments, by imposing values for the prior expectation $E[x]=S / R$ and variance $V[x]=S / R^{2}$.

In absence of prior information, it is best to adopt an objective prior. The reference prior for the Poisson model coincides with Jeffreys' prior, which is the limiting case of a Gamma function with shape parameter $S=1 / 2$ and rate parameter $R=0$. Hence we use here the (improper) Jeffreys' prior $\pi(B)=B^{-1 / 2}$ and find the (properly normalized) density which represent the solution of Bayes' formula 3 :

$$
p(B \mid k)=\mathrm{Ga}\left(B \mid k+\frac{1}{2}, 1\right)
$$

This is the reference posterior for the background in the off-source region, and the same solution is used in MK20145

Our goal is to estimate the source intensity $s$ in the target region. We use the Bayes' theorem

\footnotetext{
${ }^{4}$ The fact that the parameters assume new values well reflects the interpretation of Bayes' theorem as a way of updating our knowledge.

${ }^{5}$ The constant at the numerator of eq. (14) in MK2014 is irrelevant, as an improper prior can have any scaling factor. Here we took the latter to be one, for simplicity. What matters is that the posterior is a proper density, as is the case with eq. 5) above.
}

to write the joint posterior density in the source region as

$$
p(s, b \mid n) \propto \operatorname{Poi}(n \mid s+b) \pi(s) \pi(b)
$$

Later, we will integrate over $b$ to find the marginal posterior density $p(s \mid n)$. Hence we need to find the prior $\pi(b)$ by translating the background estimate in the control region into a background estimate in the target region. In order to do so, we use the posterior density $p(B \mid k)$ from eq. (5) to determine the prior for the background contribution $b$ in the source region. With the change of variable $b=\rho B$ we find

$$
\pi(b)=\mathrm{Ga}\left(b \mid k+\frac{1}{2}, \frac{1}{\rho}\right)
$$

It is interesting to note that the expected background in the source region is $E[b]=\rho\left(k+\frac{1}{2}\right)$ with the most probable value $\rho\left(k-\frac{1}{2}\right)$ being the mode of $\pi(b)$ when $k \geq 1$ (if $k=0$ the prior peaks at zero). The commonly used maximum likelihood estimator $\hat{b}=\rho k$ (Li\&Ma 1983) is just in between the peak value and the expected background. The background variance $V[b]=\rho^{2}\left(k+\frac{1}{2}\right)$ is also very similar (but not identical) to the commonly used value of $\rho^{2} k$.

The next step is to write down the prior for the source strength $s$. We assume no prior knowledge here, hence adopt the reference prior calculated in DC2012. The starting point for determining $\pi(s)$ is the marginal model

$$
P(n \mid s)=\int \operatorname{Poi}(n \mid s+b) \pi(b)
$$

which is in our case

$$
P(n \mid s)=\left(\frac{1}{1+\rho}\right)^{k+1 / 2} e^{-s} f\left(s ; n, k+\frac{1}{2}, \frac{1}{\rho}\right)
$$

The polynomial

$f(x ; n, c, d)=\sum_{m=0}^{n}\left(\begin{array}{c}c+m-1 \\ m\end{array}\right) \frac{x^{n-m}}{(n-m) !(1+d)^{m}}$

is a function of the real variable $x \geq 0$ with integer parameter $n \geq 0$ and real parameters $c, d>0$ whose properties are studied in DC2012.

From the marginal model, DC2012 finds the 
Fisher's information, which in our case reads

$$
\begin{aligned}
I(s) & =\left(\frac{1}{1+\rho}\right)^{k+1 / 2} e^{-s} \times \\
& \times \sum_{m=0}^{\infty} \frac{\left[f\left(s ; m, k+\frac{1}{2}, \frac{1}{\rho}\right)\right]^{2}}{f\left(s ; m+1, k+\frac{1}{2}, \frac{1}{\rho}\right)}-1
\end{aligned}
$$

The resulting reference prior is proportional to $|I(s)|^{1 / 2}$ and is improper. Hence it is defined apart from a multiplicative constant. Making use of this degree of freedom, the expression proposed by DC2012 is

$$
\pi(s)=\frac{|I(s)|^{1 / 2}}{|I(0)|^{1 / 2}}
$$

which is a monotonically decreasing function of $s$ with maximum at one for $s=06$ In the limit of perfect prior background knowledg $\varnothing^{7} \pi(b)=$ $\delta\left(b-b_{0}\right)$, one gets Jeffreys' prior for the offset-ed variable $s^{\prime}=s+b_{0}$ :

$$
\pi(s) \longrightarrow \sqrt{\frac{b_{0}}{s+b_{0}}} \equiv \pi_{0}(s)
$$

where $b_{0}=E[b]$ is a known value.

As it is shown in Appendix B, the limiting prior $\pi_{0}(s)$ can often be used in place of the more complicate reference prior $\pi(s)$ given in 111. When this is not the case, a closed-form function provides an excellent approximation of the reference prior $\pi(s)$ as described by Casadei (2014). This considerably simplifies the computation, although it is not used in this paper.

The marginal reference posterior for the source strength in the source region is finally

$$
p(s \mid n) \propto e^{-s} f\left(s ; n, k+\frac{1}{2}, \frac{1}{\rho}\right) \pi(s) .
$$

obtained after having removed the inessential constant factor $(1+\rho)^{-k-1 / 2}$ in front of the marginal model (8), as the normalization of the marginal posterior is found by dividing by the integral from zero to infinity of the expression above (which is integrable for all possible values of the background shape and rate parameters).

\footnotetext{
${ }^{6}$ Incidentally, this makes it easy to compare it to the uniform prior, a very common (although mathematically ill-defined) choice with a long tradition.

${ }^{7} \mathrm{Ga}(x \mid c, d) \rightarrow \delta\left(x-b_{0}\right)$ in the limit $c, d \rightarrow \infty$ while keeping $b_{0}=c / d$ constant.
}

The marginal reference posterior 13 is the full solution of our inference problem. Very often, it will be summarized by providing only few figures of merit, like the most probable value or the posterior expectation, plus an interval enclosing the true value with some predefined posterior probability. To complement this solution, one can check whether the counts $n$ in the source region are compatible with the background-only hypothesis by comparing $n$ with the expectation $E[b]=\rho\left(k+\frac{1}{2}\right)$ from a simple Poisson process in which no source is present.

The statistical significance $z$ quantifies the deviation between observed counts and expected background in terms of the displacement from the peak of a normal distribution in units of standard deviations. An excess of counts for which $z=3$ is commonly called "a 3 -sigma excess" and considered a real effect (that is an evidence for an additional contribution on top of the events expected from the background-only hypothesis), although more stringent requirements may be preferred, like the "5-sigma" excess traditionally required in high-energy physics to claim the discovery of a new particle, and required by MK2014 too 8

A deviation from the expected background can occur in two directions: as an excess of counts when $n>E[b]=\rho\left(k+\frac{1}{2}\right)$, or as a deficit when $n<E[b]$. The commonly used expression for the significance is eq. (17) of Li\&Ma (1983) (LM1983 hereafter). Such formula gives always a positive value for $z$, while it is more appealing to differentiate between excess and deficit of observed events with respect to the expected background. In addition, strictly speaking that formula is valid only asymptotically, although it was shown to behave well already with moderately small values of $n$.

We compute the significance of the deviation from $E[b]$ with the inclusion of the uncertainty on the background in the source region, represented by the square root of the prior background vari-

\footnotetext{
${ }^{8}$ Strictly speaking, an excess with $z=3$ or $z=5$ leads to the rejection of $H_{0}$ with a very low probabiliy of false rejection. It does not automatically imply that $H_{1}$ is true, as there could be more alternative hypotheses in competition, like e.g. an instrumental effect. However in the simplified setup in which there are only two alternatives, the excess is usually taken as evidence for the source without further discussion. We follow this tradition here.
} 
ance $V[b]$, as described by Choudalakis\&Casadei (2012) (CC2012 in the following) $]^{9}$ A Poisson process with uncertain parameter, whose probability density is represented by a Gamma distribution, is described by the Poisson-Gamma mixture

$$
\begin{aligned}
P(n \mid c, d) & =\int_{0}^{\infty} \operatorname{Poi}(n \mid b) \operatorname{Ga}(b \mid c, d) \mathrm{d} b \\
& =\frac{d^{c}}{\Gamma(c)} \frac{\Gamma(n+c)}{n !(1+d)^{n+c}}
\end{aligned}
$$

In our case, we find $c, d$ with the method of moments, from $E[b]=c / d=\rho\left(k+\frac{1}{2}\right)$ and $V[b]=$ $c / d^{2}=\rho^{2}\left(k+\frac{1}{2}\right)$. As expected, the result is $c=k+\frac{1}{2}$ and $d=1 / \rho$, the same as in eq. (7), but eq. (14) can also be used when there are other (e.g. systematic) contributions to the background uncertainty in the target region, by applying the method of moments with the corresponding (larger) variance.

For an excess, the probability $p$ to get a deviation not smaller than the observed one is given by the sum from $n$ to infinity of (14). For a deficit, $p$ is given by the sum of the terms from 0 to $n$. Next, we compute the significance $z$ by imposing that the integral from $z$ to infinity (excess) or from minus infinity to $z$ (deficit) of a standard normal distribution is equal to $p$.

This definition of $p$-value (hence of statistical significance $z$ ) is similar to the usual (frequentist) definition, with the exception that the PoissonGamma mixture (the marginal model given $H_{0}$ ) is used in place of the Poisson distribution, to which it reduces in case of negligible background uncertainty. Hence it represents a sort of hybrid computation of the probability that the data deviate from the expectation at least as much as in the actual observation. The result is valid for any value of $n$, even when $n=0$, hence it does not rely on asymptotic properties like the LM1983 significance. On the other hand, MK2014 defines the $p$-value as the probability of the model $H_{0}$ given the data [eq. (27) of MK2014]. Hence his significance (denoted by $S_{b}$ ) has a different meaning from the significance used here and by LM1983, despite from the similarity between the numerical values.

${ }^{9} \mathrm{C} / \mathrm{C}++$ macros freely available on http://svn.cern.ch/ guest/psde/ based on the ROOT framework (Antcheva et al. 2009).

\section{Results}

We apply the methods described above on the same input data as MK2014, to make a detailed comparison with that solution. Table 3 shows gamma-ray burst (GRB) data collected by FermiLAT (Abdo et al. 2009) and VERITAS (Acciari et al. 2011). Such GRBs were selected by MK2014 because they have low counts (otherwise the difference with respect to asymptotic formulae is difficult to notice): at least one among $n$ and $k$ is not bigger than 15 counts.

The first step is to estimate the background $B$ in the off-source regions with the help of eq. (5). The reference posterior for $B$ only depends on the off-source counts $k$, hence it is the same for GRBs 080607 and 090418A (both with $k=16$ ), and for GRBs 081024A and 090429B (both with $k=7$ ). Figure 1 shows all the reference posteriors for the background $B$ in the off-source regions. In addition to the counts in the off-source region, for each GRB the reference posterior mean and standard deviation of $B$ are reported, which may be useful summaries for back-of-the-envelope computations.

Next, one finds the background prior for $b=\rho B$ in the source region from eq. (7). Because the value of $\rho$ differs in each pair of GRBs with the same off-source background estimate, their background priors in the source regions are all distinct Gamma densities.

Once the reference prior from eq. (11) is computed, the final solution is provided by the (marginal) reference posterior for the source strength $s$ in the source region, eq. (13). It is worth noticing that in all cases considered here, the limiting prior $\pi_{0}(s)$ defined in (12) works equally well. There would be no relevant change if $\pi_{0}(s)$ were used in place of the reference prior $\pi(s)$ defined in (11), although the latter was used here.

Figure 2 shows the reference posteriors for $s$ for all GRBs listed in table 3, where the posteriors are summarized by reporting the HPD credible intervals with $99 \%, 95 \%, 90 \%, 68.3 \%$ posterior probability, the source intensity expectation $(E)$, median $(M)$, mode $(P)$, plus variance $(V)$, skewness $(S)$, and excess kurtosis $(K)$. Two decimal places are shown in the table, even though they do not bring any insight on the physics, because the goal is to compare against MK2014 results (where only the $99 \%$ upper bounds are reported). 
TABLE 1

GAMMA-RAY BURST DATA FROM FERMi-LAT AND VERITAS.

\begin{tabular}{|c|c|c|c|c|c|c|c|c|c|c|c|c|c|c|c|c|c|c|c|}
\hline GRB & $k$ & $n$ & $\rho$ & L99 & L95 & L90 & L68 & $E$ & $M$ & $P$ & R68 & R90 & R95 & R99 & $V$ & $S$ & $K$ & $\lambda_{99}$ & $\lambda_{99}^{\mathrm{R}}$ \\
\hline 070419A & 14 & 2 & 0.057 & 0.02 & 0.09 & 0.17 & 0.51 & 1.92 & 1.57 & 0.71 & 3.35 & 4.91 & 5.82 & 8.05 & 2.32 & 1.40 & 2.80 & 6.88 & 7.34 \\
\hline 070521 & 113 & 3 & 0.057 & 0.00 & 0.00 & 0.00 & 0.00 & 1.42 & 1.02 & 0.00 & 1.67 & 3.26 & 4.20 & 6.59 & 1.83 & 1.79 & 4.58 & 6.12 & 3.52 \\
\hline $070612 \mathrm{~B}$ & 21 & 3 & 0.066 & 0.03 & 0.13 & 0.24 & 0.69 & 2.39 & 2.01 & 1.12 & 4.08 & 5.84 & 6.83 & 9.15 & 3.17 & 1.24 & 2.13 & 8.00 & 8.54 \\
\hline 080310 & 23 & 3 & 0.128 & 0.00 & 0.00 & 0.00 & 0.00 & 1.87 & 1.45 & 0.00 & 2.26 & 4.07 & 5.09 & 7.46 & 2.58 & 1.49 & 3.08 & 7.16 & 7.08 \\
\hline 080330 & 15 & 0 & 0.123 & 0.00 & 0.00 & 0.00 & 0.00 & 0.88 & 0.60 & 0.00 & 1.01 & 2.08 & 2.77 & 4.93 & 0.81 & 2.10 & 6.74 & 4.10 & 2.40 \\
\hline 080604 & 40 & 2 & 0.063 & 0.00 & 0.00 & 0.00 & 0.00 & 1.49 & 1.11 & 0.00 & 1.77 & 3.34 & 4.25 & 6.51 & 1.85 & 1.67 & 3.99 & 6.12 & 5.66 \\
\hline 080607 & 16 & 4 & 0.112 & 0.04 & 0.19 & 0.35 & 0.95 & 2.93 & 2.55 & 1.71 & 4.90 & 6.83 & 7.89 & 10.30 & 4.17 & 1.09 & 1.62 & 9.17 & 9.83 \\
\hline $080825 \mathrm{C}$ & 19 & 15 & 0.063 & 5.95 & 7.51 & 8.39 & 10.36 & 14.27 & 13.94 & 13.28 & 18.17 & 21.27 & 22.90 & 26.29 & 15.58 & 0.50 & 0.38 & & \\
\hline $081024 \mathrm{~A}$ & 7 & 1 & 0.142 & 0.00 & 0.00 & 0.00 & 0.00 & 1.26 & 0.93 & 0.00 & 1.49 & 2.85 & 3.66 & 5.75 & 1.38 & 1.77 & 4.63 & 5.29 & 5.19 \\
\hline $090418 \mathrm{~A}$ & 16 & 3 & 0.123 & 0.02 & 0.09 & 0.17 & 0.54 & 2.15 & 1.75 & 0.57 & 3.77 & 5.52 & 6.52 & 8.94 & 2.94 & 1.34 & 2.48 & 7.64 & 8.01 \\
\hline 090429B & 7 & 2 & 0.106 & 0.02 & 0.09 & 0.18 & 0.53 & 1.95 & 1.60 & 0.76 & 3.39 & 4.96 & 5.86 & 8.08 & 2.35 & 1.38 & 2.74 & 6.92 & 7.41 \\
\hline 090515 & 24 & 4 & 0.126 & 0.02 & 0.09 & 0.19 & 0.51 & 2.36 & 1.93 & 0.52 & 2.88 & 6.05 & 7.13 & 9.68 & 3.54 & 1.30 & 2.27 & 8.34 & 8.66 \\
\hline
\end{tabular}

Note.- Low count gamma-ray burst data where either or both of $n$ or $k$ are $\leq 15$. All data report VERITAS measurements (Acciari et al. 2011), apart from GRB 080825C, detected by Fermi-LAT (Abdo et al. 2009). The posterior HPD credible intervals are reported with $99 \%, 95 \%, 90 \%, 68.3 \%$ posterior probability, together with source intensity expectation $(E)$, median $(M)$, mode $(P)$, variance $(V)$, skewness $(S)$, and excess kurtosis $(K)$. The last two columns report the $99 \%$ upper bounds computed by MK2014 using his solution and the method by Rolke et al. (2005). GRB 080825C is the only clear detection: we obtain $s=13.28_{-2.92}^{+4.89}$, MK2014 obtains $13.28_{-3.49}^{+4.16}$, and 13.7 is the official result by the Fermi-LAT collaboration.

As remarked by MK2014, the only clear detection is GRB 080825C, observed by Fermi-LAT (Abdo et al. 2009): we obtain $s=13.28_{-2.92}^{+4.89}$ with significance $z=6.26$, whereas MK2014 obtains $s=13.28_{-3.49}^{+4.16}$ with significance $S_{b}=6.11$ computed by mapping the posterior probability of $H_{0}$ onto a Gaussian metric. Both Bayesian solutions find the same peak value for the source strength, which is only $3 \%$ weaker than the result of 13.7 units obtained by the Fermi-LAT collaboration, a difference ten times smaller than the standard deviation computed here $(\sqrt{V}=3.95)$, hence negligible. Our result is slightly more suggestive of higher source counts than MK2014, as the rightasymmetry of our $68.3 \%$ credible interval is more pronounced. This implies that our source strength expectation (14.27 units) should be larger than MK2014 (where this value is not reported). However, this small difference is of little practical importance.

Apart from 080825C, all other GRB data do not show any evidence for a detectable source, as the significance values reported in figure 3 confirm. For GRBs 070521, 080310, 080330, 080604 and $081024 \mathrm{~A}$, the reference posterior is monotonically decreasing with maximum probability density at zero: the right edges of their HPD intervals are all upper bounds to the source strength. Although the posteriors of the other GRBs are not monotonically decreasing functions, their peaks are so near to zero that one has in practice upper bounds also in these cases. The significance $z$ of the deviation from the background-only expectation is 0.83 for 070419A, 0.93 for 070612B, 1.14 for 080607, 0.44 for $090418 \mathrm{~A}, 0.87$ for $090429 \mathrm{~B}$, and 0.33 for 0900515: clearly $H_{0}$ can not be rejected. In addition, their posterior 99\% HPD intervals, when keeping a single decimal place, all start at zero. In conclusion, there is no clear evidence for some additional contribution in addition to the photon counts expected from the background alone.

It is interesting to look at the distribution of the significance $z$ of the deviations between observed counts and expected background, as this is a quick way of checking that the background-only hypothesis is valid for the entire set of measurements. By definition, under repeated sampling the model $H_{0}$ will give values of $z$ which are normally distributed with center at zero and unit standard deviation.

A plot produced with all GRBs listed in table 3 apart from GRB 080825C is shown in figure 3 . The values computed according to LM1983 are also shown for comparison. As mentioned above, they are always positive, which makes a difference when a deficit of events is observed. However, this case is less interesting than the observation of an excess of counts, for which the agreement is acceptable. The formula by LM1983 overestimates the significance when the latter is small (by $15 \%$ when $z \approx 1$, increasing when $z \rightarrow 0$ but decreasing when $z$ increases), which is not a big problem in practice. When the significance is high, it gives 


\section{On/off measurements: background posteriors in the off-region}

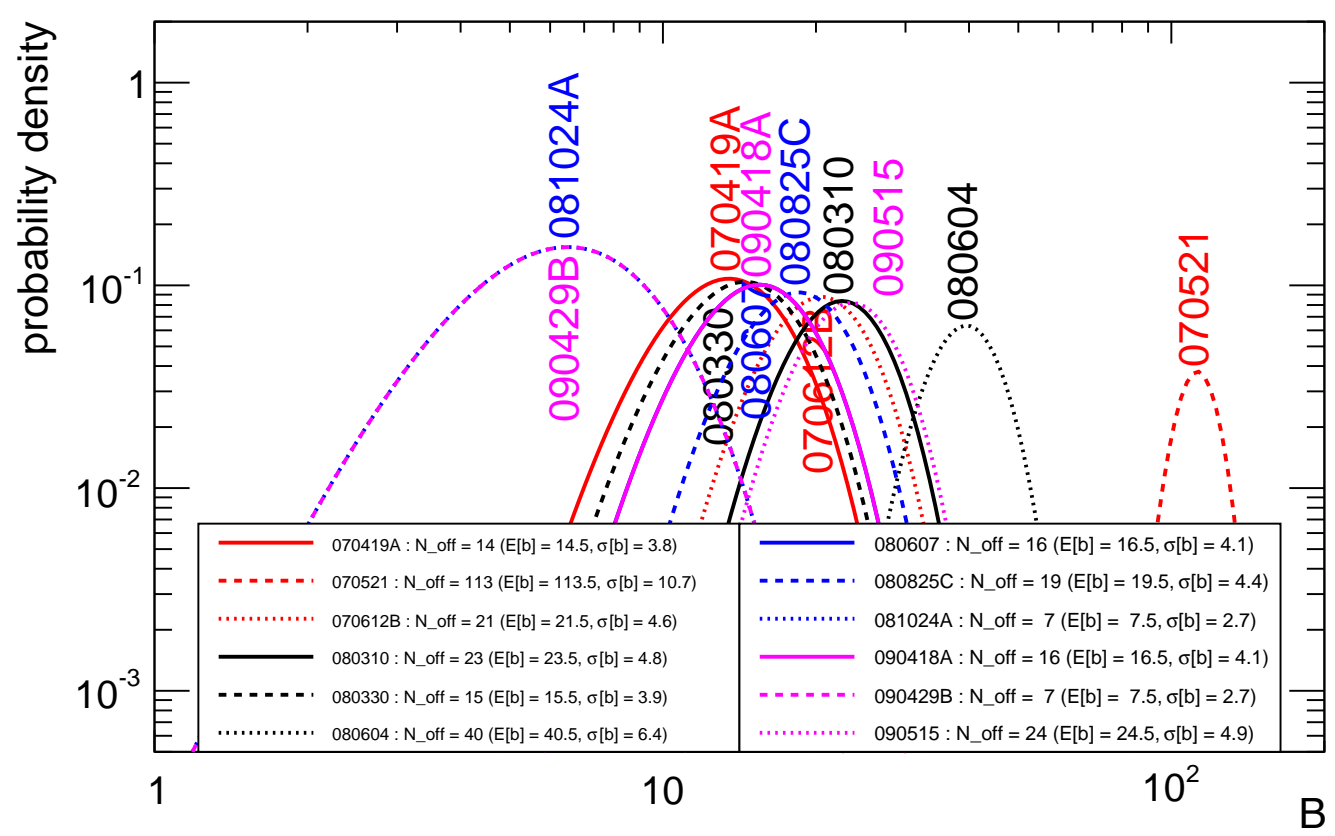

Fig. 1.- Reference posteriors for the background in off-source regions. The GRB name is followed by the counts in the off-source and source regions, and by the resulting posterior mean and standard deviation.

very similar results to our approach. For example, the detection of GRB $080825 \mathrm{C}$ by Fermi-LAT has significance $z=6.2610$ The formula by LM1983 gives a value of 6.36 while MK2014 obtains 6.11, hence the three methods agree that the result is very significant. The good agreement $(1.6 \%)$ between the standard formula of LM1983 and our method is connected to their very similar background uncertainty estimates $(\rho \sqrt{k}$ for the standard method of LM1983 and $\rho \sqrt{k+1 / 2}$ in our case). However this uncertainty is purely statistical: if any additional contribution exists, then the formula by LM1983 is not able to account for it and the method by CC2012 should be used instead, as it is more general (the uncertainty is not assumed but is an input parameter). Another possibility is to compute the posterior probability of $H_{0}$ given the data, and map it onto a Gaussian metric. This is what is done by MK2014, whose result is a bit (2.4\%) smaller than, but still appreciably similar to ours, despite from the different

${ }^{10}$ For illustration purposes only, figure 3 also shows than one finds $z=6.80$ when ignoring the background uncertainty. meaning.

By collecting all significance values one creates the "pull distribution", which in case of purely stochastic fluctuations should follow a standard normal distribution (as it is indeed the case when simulating a large number of pseudo-experiments). The inset at the top-left corner of figure 3 shows that the GRB measurements - with the exception of GRB 080825C, detected with more than sixsigma statistical significance and not shown there - do not show any strong deviation from that distribution (dashed black curve), which would suggest that $H_{0}$ does not all for the entire sample. Although a Gaussian fit (red curve) actually confirms the preference for positive fluctuations which is visible in the bottom plot, it also says that the results are more tightly clustered than expected. The shift of the barycenter is not significant, being about twice as big as the uncertainty on its position, confirming that the null hypothesis of pure background counts well describes the set of observations. 


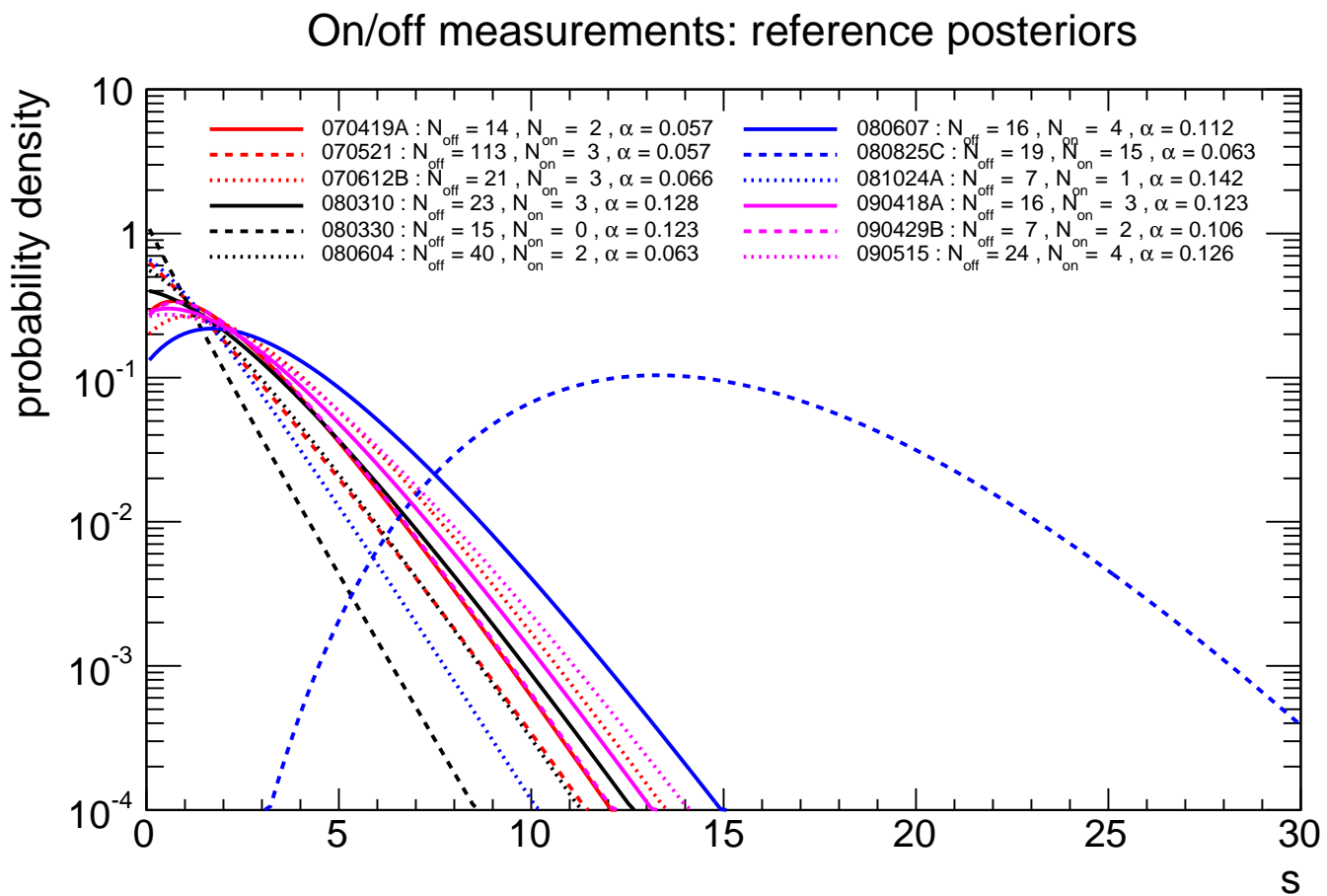

Fig. 2.- Marginal posteriors for GRB data. The GRB name is followed by the counts in the off-source and source regions, and by the ratio between the background fluxes in these regions.

\section{Summary and discussion}

We have illustrated how the objective Bayesian solution to the inference problem for the $\operatorname{Poi}(s+b)$ model can be applied to the on/off problem. Our solution is the marginal reference posterior probability density for the source strength $s$, given the measured counts $n$ in the source region, and the auxiliary measurement of background-only counts summarized by the off-source counts $k$ and the ratio $\rho$ between the background fluxes in the two regions. Based on the reference prior computed by DC2012, this solution appears to be more conservative (higher upper bounds) when there is no clear detection of additional photons with respect to the background-only expectation in the source region, compared to the posterior proposed recently by MK2014 and to the frequentist method based on asymptotic properties of the profile likelihood test statistic by Rolke et al. (2005).

The approach by MK2014 also aims at providing an objective Bayesian result. Its most delicate point is the choice of the prior for the source region. The choice of Jeffreys' prior in the $(s, b)$ space may give troubles which can be avoided if one consider the marginal model instead (obtained by integrating over the entire range of $b$, weighted by its prior). On the other hand, the marginal model is 1-dimensional and the corresponding reference prior is known. Reference priors, when available, are the recommended objective priors in the statistics literature, as they possess a number of desirable properties and are well "calibrated" from the frequentist point of view. In 1-dimensional problems, they usually coincide with Jeffreys' priors, but this is not true in multidimensional problems.

Another possible source of troubles is the hypothesis testing step in the method proposed by MK2014, as the improper priors used both in the off-source and source regions are not identical. MK2014 proposes an ad-hoc procedure to overcome the problem, which is based on the questionable assumption that measuring $k=0$ and $n=0$ does not change our degree of belief about the two alternative hypotheses. We propose to avoid the problem by omitting the comparison between $H_{0}$ and $H_{1}$. One can compute the statistical 


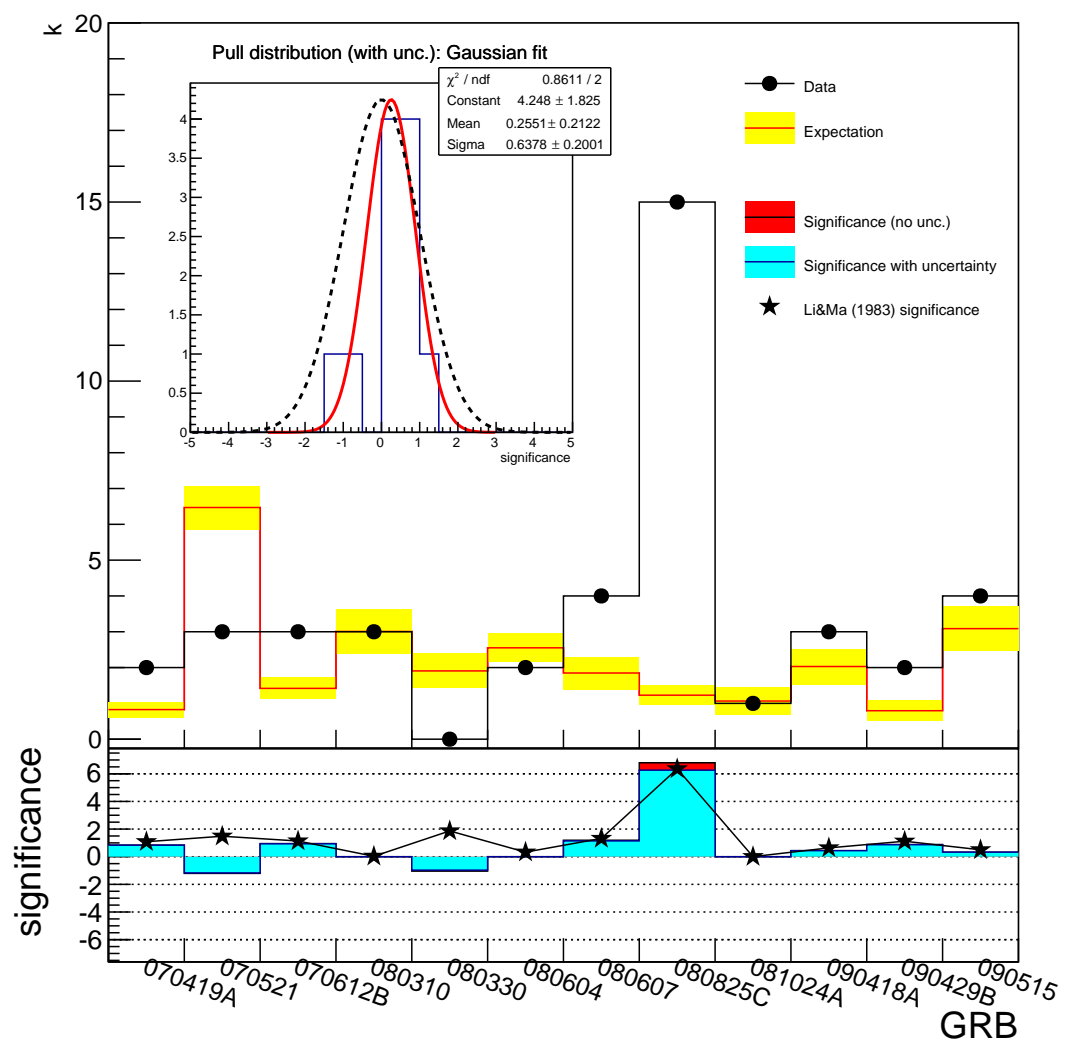

Fig. 3. - The observed counts (full dots) in the source region of each GRB are compared to the expected background and its uncertainty (red histogram with yellow bands representing fluctuations of one standard deviation in both directions). The plot at the bottom shows the significance of the deviation in each bin, computed with (cyan histogram) and without (red histogram) accounting for the uncertainty on the background. The black stars correspond to the significance computed accordingly to Li\&Ma (1983). The inset in the top-left corner shows the pull distribution computed with background uncertainties, with a Gaussian fit (red line). A standard normal distribution (centered at zero with unit standard deviation) is also shown for comparison (dashed black line).

significance of the deviation between the observed counts and the background-only expectation on the basis of $H_{0}$ alone, as a conservative check that the probability of claiming a false detection is low enough. This complements the estimate of the source strength $s$ provided by the marginal reference posterior.

In case the hypothesis test is considered a fundamental step (which seems not to be the case here), it might be worth noticing that Bernardo (2011) recommends to base our decision on the comparison between the reference posteriors of each model by means of an invariant information- based loss function (the "intrinsic discrepancy"). This promises to be the best way of achieving an universally applicable procedure which guarantees objective decisions. Unfortunately, this approach to the Bayesian hypothesis testing is not yet widespread in the scientific community, where most people continue to look at the Bayes factors (ill-defined in our case; see also the discussion in Bayarri et al. (2008)).

The HPD intervals chosen by MK2014 cover $99 \%$ posterior probability. In other words, they are the shortest $99 \%$ credible intervals on the source strength $s$, given the measurements in the 
on/off regions. Even though they are not invariant under reparametrization, nor it is the most probable value, there is little or no discussion in the astrophysics community about the best choice for the parameter of interest: everybody just looks at $s$. Hence here we also show the posterior mode with $99 \%$ credible HPD intervals, although one might consider more complicate ways of summarizing the result in an invariant way. The interested reader can find details about the "reference posterior intrinsic loss" function which allows one to find the "intrinsic estimator" of the parameter of interest, together with "intrinsic $99 \%$ credible regions" for $s$, in Bernardo (2005b) and Bernardo (2007).

The numerical comparison with the results obtained by MK2014 shows that the two methods are in decent agreement, although the use of Jeffreys' prior leads to narrower posterior densities than the marginal reference posterior. This means that the upper bounds obtained by MK2014 are always tighter than those obtained here.

The only clear case of unambiguous GRB detection by is $080825 \mathrm{C}$ by Fermi-LAT (Abdo et al. 2009). For this GRB, MK2014 obtains $s=$ $13.28_{-3.49}^{+4.16}$ whereas the result obtained here is $s=13.28_{-2.92}^{+4.89}$ : even though the posterior peak is at the same position as in MK2014, our result is slightly more suggestive of a higher intensity, although it well overlaps with MK2014 within the uncertainties.

With all GRB data considered here, the very simple approximate reference prior $\pi_{0} \propto(s+$ $E[b])^{-1 / 2}$ provides practically the same result as the (more complicate) reference prior. This is always true when the rate parameter describing the Gamma prior for $b$ is large enough (in practice, it is sufficient to be larger than a few units), or when the shape parameter is large. In our case, the shape parameter of the background prior in the source region is $S=k+\frac{1}{2}$, while the rate parameter is $R=\frac{1}{\rho}$. The approximate reference prior $\pi_{0}(s)$ differs less than $1 \%$ from the reference prior when $R>4$ or $S>40$, plus a portion of the parameters space which does not satisfy any of these requirements (Appendix B). The first condition is fulfilled by all GRBs in table 3 apart from GRBs $080310(S=23.5, R=7.8), 081024 \mathrm{~A}(S=$ $7.5, R=7.04)$, and $090515(S=24.5, R=7.9)$. However these three GRBs have shape and rate parameters which fall in regions of the parameters space in which $\pi_{0}(s)$ differs very little from the reference prior. This means that the approximate marginal reference posterior

$$
p_{0}(s \mid n) \propto \mathrm{Ga}\left(s+E[b] \mid n+\frac{1}{2}, 1\right)
$$

(Casadei 2014) could have been used in place of the marginal reference posterior (13), with a considerable simplification.

Finally, we have noticed that the significance $z$ obtained with the standard asymptotic formula by Li\&Ma (1983), when it is not too small, well agrees with the values calculated as suggested by CC2012, which are correct for any value of $n$ (including the case $n=0$ ). By treating the background uncertainty as a free parameter, the more recent definition of $z$ is more general than the standard formula and should be used whenever additional sources of uncertainty exist beyond the pure statistical fluctuations connected with the finite number of photons collected while pointing off-source.

\section{Conclusion}

The Bayesian approach proposed by MK2014 aims at providing an objective solution for the on/off measurement. Two possible sources of troubles with this approach are connected with the use of Jeffreys' prior in the bidimensional space $(s, b)$ of source and background intensities in the target region, and with the hypothesis test performed with improper priors.

Instead of using the Jeffreys prior, a different approach exists which provides an objective Bayesian solution as the marginal reference posterior for $s$ obtained after reducing the problem to a 1-dimensional problem. This is done by integrating over the background intensity $b$ in the target region, for which an informative prior exists. The reference prior for this 1-dimensional problem is known, which ensures that the solution possesses all the important features of the reference posteriors, including invariance under reparametrization and good frequentist properties. Thus, it is recommended to compute the reference posterior, possibly with the help of some approximation of the reference prior when the difference is small enough (which can be judged by checking the background parameters as explained in Appendix B. 
A formal two-steps procedure, in which the comparison between background-only $H_{0}$ and source+background $H_{1}$ hypotheses is performed before estimating the source intensity in the target region, is not strictly necessary. A simpler approach is to estimate $s$ directly, and only check the significance of the deviation from the expectation from pure background counts in case the posterior for $s$ suggests a non negligible source intensity. As the significance is computed with $H_{0}$, one avoids the complications arising when comparing two nested hypotheses whose parameters have improper priors.

The significance $S_{b}$ defined by MK2014 is Bayesian, in the sense that it corresponds to the probability that $H_{0}$ is true given the data. Thus, it is conceptually different from the usual definition of statistical significance $z$, in terms of the probability of obtaining data with deviations at least as large as the observed one, given $H_{0}$. The latter is a frequentist concept, which is typically computed with the help of asymptotic formulae as in LM1983. Here we have adopted a hybrid approach which computes the probability of deviations given $H_{0}$ with the marginal model obtained after integrating over the background in the target region, given by a Poisson-Gamma mixture. This approach is superior to LM1983 for three reasons: it does not rely on asymptotics, it differentiates between excess and deficit, and it allows to include any uncertainty on the background (while LM1983 only account for the statistical uncertainty from the auxiliary measurement). As expected, when the significance is high enough, the three definitions provide results which are numerically very similar. However, our approach behaves differently for mildly significant results, for which LM1983 tend to overestimate $z$, and for all other cases (including deficits, which get assigned positive $z$ values by LM1983). 


\section{A. Upper limits on detectable sources}

Kashyap et al. (2010) emphasize the important difference between the upper bound on the source intensity $s$ in the target region, given the observed number $n$ of counts and the prior knowledge about the background intensity $b$ in the same region, and the upper limit on the detectable sources with the chosen detection technique. Upper bounds (typically at $90 \%, 95 \%$ or $99 \%$ confidence level) are a way of summarizing the result of a measurement, when there is no significant evidence of an excess with respect to the counts expected from the background alone. On the other hand, upper limits are connected to the detection technique and can/should be computed before looking at the experimental outcome. In other words, upper limits are connected to the sensitivity of the chosen technique, and do not depend on the counts $n$ in the source region. However, they depend on the estimated background in the target region, which in our case means that they depend on the counts $k$ in the off-source region, on the prior knowledge about the background rate $B$ in this control region, and on the known ratio $\rho=b / B$ between the background rates in both regions. In this paper we only addressed upper bounds on the measured source intensity. Here we provide an algorithm to compute the upper limit accordingly to Kashyap et al. (2010), whose approach is generally valid for any detection technique, for the case of on/off measurements.

The notation by Kashyap et al. (2010) is the following. The source and background rates in the target region are $\lambda_{S}, \lambda_{B}$, respectively, with corresponding exposure times $\tau_{S}, \tau_{B}$. The ratio between the two regions is $r$, and one performs two measurements whose results are the counts $n_{S}$ and $n_{B}$ in the source and off-source regions, with

$$
n_{B} \mid\left(\lambda_{B}, \tau_{B}, r\right) \sim \operatorname{Poi}\left(r \tau_{B} \lambda_{B}\right) \quad \text { and } \quad n_{S} \mid\left(\lambda_{S}, \lambda_{B}, \tau_{S}\right) \sim \operatorname{Poi}\left(\tau_{S}\left(\lambda_{S}+\lambda_{B}\right)\right)
$$

The correspondence to our notation is the following:

$$
\begin{array}{lll}
n \equiv n_{S} & s \equiv \tau_{S} \lambda_{S} & B \equiv r \tau_{B} \lambda_{B} \\
k \equiv n_{B} & b \equiv \tau_{S} \lambda_{B} & \rho \equiv \tau_{S} /\left(r \tau_{B}\right)
\end{array}
$$

In addition to (i) the background prior $\pi(b)$ in the source region, an upper limit also depends on (ii) the "size of the test" $\alpha$, that is the predefined maximum tolerable probability of false detection (or Type I error rate), and on (iii) the predefined minimum "power of the test" $\beta$, which is connected with Type II errors ( $\beta=1$ - Type II error rate), happening when one makes a false exclusion (i.e. does not recognize that a source is present in the target region). Common values for $\alpha$ are 0.05, 0.003 (a "three-sigma" threshold), and 0.001 , whereas a "five-sigma" threshold would correspond to $5.7 \times 10^{-7}$. In addition, typically one sets $\beta=0.5$ or $\beta=0.9$ to require at least $50 \%$ or $90 \%$ detection probability, when speaking about the expected sensitivity of the measurement process for a given source strength.

For the on/off measurement we choose $n$ as the test statistic and fix a threshold $n_{\min } \geq 0$ such that if $n>n_{\min }$ we claim that a source has been detected, and if $n \leq n_{\min }$ we declare that the measurement is compatible with the background-only hypothesis. The threshold $n_{\min }$ is chosen such that the probability of false detection (i.e. of wrongly rejecting the null hypothesis $H_{0}$ ) does not exceed $\alpha$ :

$$
\alpha \geq P\left(n>n_{\min } \mid s=0\right)=1-\sum_{n=0}^{n_{\min }} \int \operatorname{Poi}(n \mid b) \pi(b) \mathrm{d} b=1-\frac{1}{(1+\rho)^{k+1 / 2} \Gamma\left(k+\frac{1}{2}\right)} \sum_{n=0}^{n_{\min }} \frac{\Gamma\left(n+k+\frac{1}{2}\right)}{n !(1+1 / \rho)^{n}}
$$

where we used the Poisson-Gamma mixture from eq. 14 with $c=k+\frac{1}{2}$ and $d=1 / \alpha$, i.e. we used the background prior $\pi(b)$ from eq. (7). Because the threshold $n_{\min }$ changes at discrete steps, the actual Type I error rate will typically be smaller than $\alpha$.

The expected detection probability when $s>0$ is

$$
\begin{aligned}
\beta(s \mid \alpha) & =P\left(n>n_{\min } \mid s\right)=1-\sum_{n=0}^{n_{\min }} \int \operatorname{Poi}(n \mid s+b) \pi(b) \mathrm{d} b=1-\frac{e^{-s}}{(1+\rho)^{k+1 / 2}} \sum_{n=0}^{n_{\min }} f\left(s ; n, k+\frac{1}{2}, \frac{1}{\rho}\right) \\
& =1-\frac{e^{-s}}{(1+\rho)^{k+1 / 2} \Gamma\left(k+\frac{1}{2}\right)} \sum_{n=0}^{n_{\min }} \sum_{m=0}^{n} \frac{\Gamma\left(m+k+\frac{1}{2}\right) s^{n-m}}{m !(n-m) !(1+1 / \rho)^{m}}
\end{aligned}
$$


obtained after inserting the polynomial (9) into the marginal model (8). For $s \rightarrow 0$ eq. (A4) gives the last expression of (A3), because only the term with $m=n$ survives in the second sum. Thus $\beta(0 \mid \alpha) \leq \alpha$, which means that one must find a compromise, while aiming at a low probability of claiming a fake source and a high probability to detect a true but faint source.

Now that all ingredients are available, we can formulate the algorithm for computing the upper limit. Once the size and power of the test have been chosen ( background in the target region, where the prior $\pi(b)$ shall be chosen as a Gamma density whose parameters are determined by the method of moments, starting from the background expectation and variance. In the on/off measurement, we use the control region to find the background in the target region, given by eq. (7). Next, we define a threshold for detecting the source, in terms of the minimum number $n_{\min }$ of counts which ensures that our Type I error rate does not exceed $\alpha$, following the inequality (A3). Finally, one takes as the upper limit the smallest value of $s$ for which $\beta(s \mid \alpha) \geq 0.5$, where $\beta(s \mid \alpha)$ is computed in eq. (A4).

\section{B. Approximate forms for the reference prior}

Here we report useful approximations to the reference prior $\pi(s)$ defined in eq. (11), from Casadei (2014). A movie comparing the reference prior with these approximations and with the flat prior is available on https://www.youtube.com/watch?v=vqUnRrwinHc, clearly showing when different approximations should be used.

The limiting form $\pi_{0}(s)$ of the reference prior when there is certain knowledge of the background in the source region is given by eq. (12). The limit of perfect knowledge is approached by increasing values of the shape parameter, as the relative uncertainty on the background in the source region is $\sqrt{V[b]} / E[b]=1 / \sqrt{S}$. However, it turns out that even at small values of $S$ there are cases in which $\pi_{0}(s)$ provides a very good approximation to $\pi(s)$.

In order to quantify the deviation from $\pi(s)$, their relative RMS difference has been computed on the signal range $0 \leq s \leq 70$, by dividing the distance

$$
d_{2}=\left(\int_{0}^{70}\left[\pi_{0}(s)-\pi(s)\right]^{2} \mathrm{~d} s\right)^{1 / 2}
$$

by the integral of the reference prior over the same range.

For most practical purposes, a relative RMS difference below $1 \%$ is acceptable, as this is the order of magnitude of the maximum change in the posterior in the limit of very few or zero observed counts. For increasing $n$, the changes of the posterior become smaller and smaller. Figure 4 shows that the $\pi_{0}(s)$ is satisfactory (differing from $\pi(s)$ by less than 1\%) when the shape parameter is larger than 40 or the rate parameter is larger than 4 , and in some case even for lower values.

It should be emphasized that the threshold at $1 \%$ chosen here is arbitrary and quite conservative. In most applications larger deviations can be acceptable, as the posteriors will quickly become indistinguishable for increasing number $n$ of observed counts. In addition, the common practice is to summarize the posterior by providing one value (e.g. the expectation or the mode) and some estimate of its uncertainty (e.g. the shortest interval covering $68.3 \%$ posterior probability), by rounding the values to the minimum meaningful number of digits. Often, this summary is quite robust compared to relative RMS differences of several percent. 


\section{Relative RMS difference between $\pi(\mathrm{s})$ and $\pi_{0}(\mathrm{~s})$}

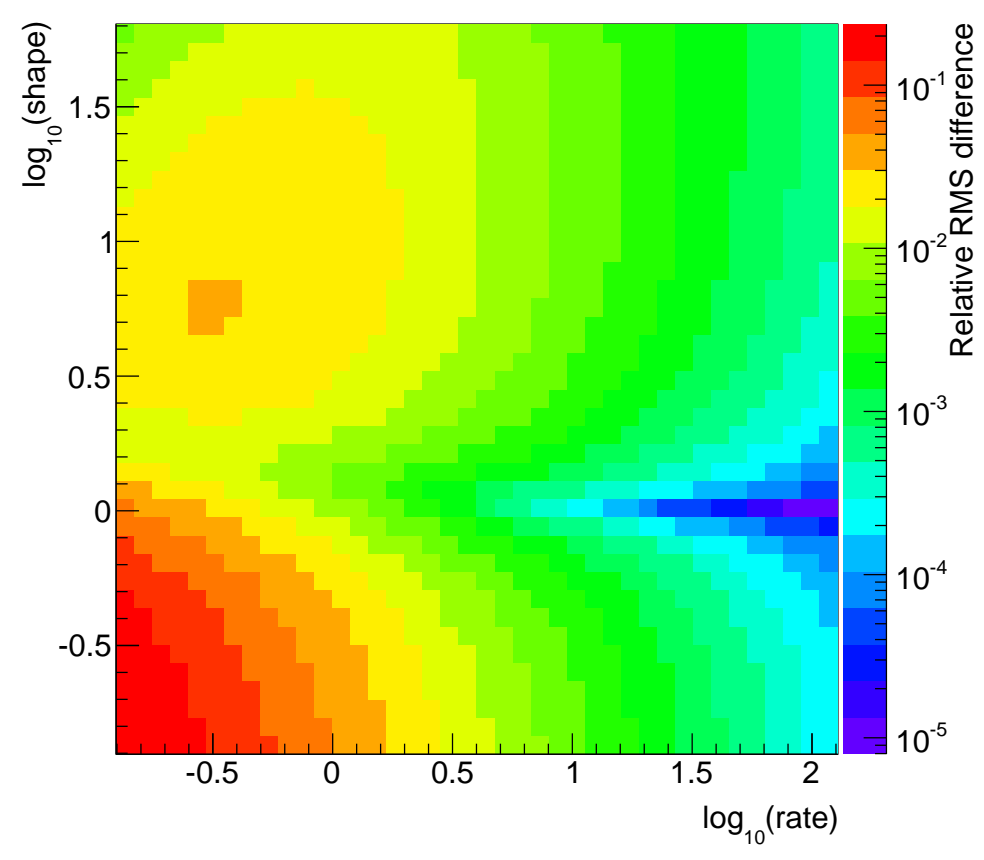

Fig. 4.- Relative RMS difference between $\pi(s)$ and $\pi_{0}(s)$ as a function of the shape and rate parameters of the background prior in the source region (Casadei 2014). 


\section{REFERENCES}

A.A. Abdo et al., FERMI observations of high-energy gamma-ray emission from GRB 080825C, ApJ707 (2009) 580, doi: 10.1088/0004-637X/707/1/580.

V.A. Acciari et al., VERITAS observations of gamma-ray bursts detected by SWIFT, ApJ743 (2011) 62, doi: 10.1088/0004-637X/743/1/62

I. Antcheva et al., ROOT - A C++ framework for petabyte data storage, statistical analysis and visualization, Computer Physics Communications 180 (2009) 2499, doi: 10.1016/j.cpc.2009.08.005

M.J. Bayarri, J.O. Berger \& G.S. Datta, Objective Bayes testing of Poisson versus inflated Poisson models, IMS Collections 3 (2008) 105, doi: 10.1214/074921708000000093

J.O. Berger, J.M. Bernardo \& D. Sun, Overall objective priors, Tech. Rep. (Duke University, USA), 2013 http://www.uv.es/ bernardo/ BBS2013.pdf.

J.M. Bernardo, Reference analysis, Handbook of Statistics 25 (D.K. Dey and C.R. Rao eds.). Amsterdam: Elsevier (2005) 17-90.

J.M. Bernardo, Intrinsic credible regions: An objective Bayesian approach to interval estimation, Test 14 (2005) 317, doi: 10.1007/BF02595408

J.M. Bernardo, Ojective Bayesian point and region estimation in location-scale models, Sort 31 (2007) 3-44.

J.M. Bernardo, Integrated Objective Bayesian Estimation and Hypothesis Testing, Bayesian Statistics 9 (2001) 1, doi: 10.1093/acprof:oso/9780199694587.003.0001.

D. Casadei, Reference analysis of the signal + background model in counting experiments, JINST 7 (2012) P01012, doi: 10.1088/1748-0221/7/01/P01012, arXiv: $1108.4270[$ DC2012].

D. Casadei, Reference analysis of the signal + background model in counting experiments II. Approximate reference prior, arXiv: 1407.5893
2014_JINST_9_T10006, doi: 10.1088/17480221/9/10/T10006

A. Caldwell, D. Kollar \& K. Kröninger, BAT - The Bayesian Analysis Toolkit, Computer Physics Communications 180 (2009) 2197 doi: 10.1016/j.cpc.2009.06.026, arXiv: 0808.2552

G. Choudalakis \& D. Casadei, Plotting the Differences Between Data and Expectation, Eur. Phys. J. Plus 127 (2012) 25, doi: 10.1140/epjp/i2012-12025-y, arXiv: 1111.2062 [CC2012].

V.L. Kashyap et al., On Computing Upper Limits to Source Intensities, Astrophys. J. 719 (2010) 900, doi: 10.1088/0004-637X/719/1/900

M.L. Knoetig, Signal discovery, limits, and uncertainties with sparse On/Off measurements: an objective Bayesian analysis, Astrophys. J. 790 (2014) 106, doi: 10.1088/0004637X/790/2/106, arXiv: 1406.2922 [MK2014].

T.-P. Li \& Y.-Q. Ma, Astrophys. J. 272 (1983) 317, doi: 10.1086/161295 [LM1983].

W.A. Rolke, A.M. López \& J. Conrad, Nucl. Instrum. Methods A 551 (2005) 493, doi: 10.1016/j.nima.2005.05.068, arXiv: physics $/ 0403059$

This 2-column preprint was prepared with the AAS LATEX macros $\mathrm{v} 5.2$ 